\title{
模拟计算纳入现代分析技术课程的初探
}

\author{
温大尉 ${ }^{*}$ \\ 五邑大学应用物理与材料学院, 广东江门529020
}

\begin{abstract}
摘要: 现代分析技术是一门讲授材料分析理论和仪器的课程。材料分析的基础理论涉及微观尺度和复杂的数理表达, 对学生而言是难点。针对这个问题, 结合了两个教学实例, 采用理论模拟和密度泛函理论进行X射线衍射谱和拉曼 光谱的分析, 利用计算机仿真模拟技术进行教学研究。结果表明, 计算机的模拟能够简单、直观和可视化地解释仪 器与材料的相互作用并分析所得数据, 为学生以后在工程或科研方面的工作夯实基础。
\end{abstract}

关键词：晶体；X射线衍射；拉曼散射；粉末衍射模拟；密度泛函理论

中图分类号: G64; O6

\section{Exploration of Introducing Simulation \& Calculation in Modern Analytical Technology Curriculum}

\author{
Dawei Wen * \\ School of Applied Physics and Materials, Wuyi University, Jiangmen 529020, Guangdong Province, China.
}

\begin{abstract}
Modern analytical technology is a curriculum of materials analysis and instrumentation. The basic theories of materials analysis contain microscale objects and complicated mathematical contents. It is uneasy for students to understand the knowledge by the traditional education method. Therefore, the simulation of powder X-ray diffraction and Raman scattering spectrum with computers serve as two teaching examples for the education research. The results demonstrate computational simulation explains the interaction of instruments and materials in an easy, intuitive and visual way, helping students in the future work of engineering or scientific research.
\end{abstract}

Key Words: Crystal; X-ray diffraction; Raman scattering; Simulation of powder diffraction;

Density functional theory

现代分析技术是一门主要面向化学、材料、物理、环境等专业硕士研究生的专业基础课或专业 选修课。以笔者所在单位为例, 现代分析技术课程作为必修课, 面向材料与化工和材料科学与工程 专业的硕士研究生。此课程主要分成原理和实操两部分, 前者讲解X射线衍射、扫描电镜、透射电 镜、紫外-可见吸收光谱、红外吸收光谱、拉曼散射光谱、核磁共振和色谱等测试分析技术, 实操则 是实际操作仪器进行测试。这些技术都是基于物质的物理或化学性质的分析手段, 通过特殊的光学 或者电子等探测方法与物质内部的原子和电子起相互作用, 定量或者定性地分析物质的成分和微 观结构。物理、化学、材料、环境等理工类研究生在完成研究课题的过程中, 往往需要用到以上技 术。比如, 材料的晶体结构和晶体表面形貌与催化活性非常相关, 主攻光催化材料的研究生常常需

收稿: 2021-07-28; 录用: 2021-08-11; 网络发表: 2021-09-13

“通讯作者, Email: ontaii@163.com

基金资助: 五邑大学高层次人才计划(2019TP007); 江门市科技项目((2017) 307,(2017) 149, (2018) 352); 广东省高等学校光电材料与应用重点实 验室(2017KSYS011); 广东省普通高校重点领域专项(新一代信息技术) (2021ZDZX1022); 五邑大学教学质量工程与教学改革工程项目(JX2019072) 
要使用X射线粉末衍射、红外、拉曼光谱和透射电镜的选区电子衍射表征催化剂的结构, 使用扫描电 镜观察材料表面形貌。另外, 掌握现代分析技术的知识, 为将来的毕业实习及作为工程师或科研人 员的就业, 提供必备素质和专项技能。因此, 现代分析技术对于基础科学的理工类研究生极为重要。

现代分析技术的测试与物质内部或表面息息相关, 在教学中涉及到原子、离子、化学键与振动、 晶体结构和数学公式(比如X射线的布拉格公式)等。这些微观概念无法直接通过肉眼观察, 在生活中 几乎无法接触到, 对学生而言过于抽象 ${ }^{[1,2]}$ 。比如, 教学中的一个突出问题是物质结构与测试结果的 逻辑关系: 物质结构的微小变化, 会导致测试结果如何变化? 反过来测试结果的差异意味着物质结 构发生怎样的变化? 因此, 传统的教学模式难以满足教学需求, 需要研究新型的教学方法。20-21世 纪计算机无论在硬件还是软件方面, 发展都非常迅猛, 为材料的谱学模拟提供了可能。通过晶体学 和密度泛函理论软件, 可以根据晶体微观结构, 模拟实验测试结果。如果修改了晶体的参数或者原 子位置, X射线衍射和拉曼光谱的发射峰位置和强度都会发生相应的变化, 这样结构与测试结果的 联系, 可以通过计算机 “实时” 观察到, 可以使师生都产生参与感 ${ }^{[3]}$ 。本文通过 2 个教学实例, 利用 晶体学软件和第一性原理计算软件, 把抽象的晶体学概念以生动形象的形式呈现出来, 帮助学生建 立现代分析技术的知识框架。

\section{1 使用 Rietan FP模拟 $\mathrm{Na}_{1-x} \mathrm{~K}_{x} \mathrm{Cl}$ 的X射线粉末衍射谱}

$X$ 射线衍射基于布拉格公式，其中 $d$ 为晶面距， $\lambda$ 为 $X$ 射线的波长:

$$
2 d \sin \theta=n \lambda \cdot n=1,2, \cdots
$$

可见, 晶面距如果变大, 则对应的衍射角会下降。但是, 数学表达对学生而言不容易理解, 而 且没有X射线衍射谱作为例子, 过于抽象, 很难给学生留下深刻的印象。因此, X射线粉末衍射谱的 讲解结合软件模拟, 进行如下设计:

(1) 选择结构相对简单的物质进行模拟, 学生容易理解物质内部的变化;

(2) 选择生活中较为常见的物质, 增加学生的兴趣, 降低学生的心理门槛;

(3) 该物质能形成固溶体, 晶面距 $d$ 发生变化最终能影响衍射图谱;

(4) 模拟一系列固溶体, 并观察衍射峰位与晶胞参数的关系;

(5) 使用免费软件Rietan FP, 可以完全避免版权纠纷, 只需要引用原作者的论文即可(Rietan FP 是日本国立材料研究所研发的软件包, 主要用于X射线衍射或者中子衍射的精修和图谱模拟) ${ }^{[4]}$ 。

根据以上设计理念, 选择食盐与氯化钾 $(\mathrm{NaCl}-\mathrm{KCl})$ 固溶体, 符合结构简单、生活中常见的原则。 $\mathrm{NaCl}-\mathrm{KCl}$ 固溶体为立方晶系, 空间群为 $F m \overline{3} m$, 金属离子处于晶胞的面心和顶角, $\mathrm{Cl}^{-}$阴离子位于每 条棱的中央, $\mathrm{NaCl}$ 和 $\mathrm{KCl}$ 混溶得 $\mathrm{Na}_{1-x} \mathrm{~K}_{x} \mathrm{Cl}$ (图1(a)), 其中金属离子 $\mathrm{Na}^{+}$和 $\mathrm{K}^{+}$均与周围 6 个 $\mathrm{Cl}^{-}$离子配位, 形成八面体。由于 $\mathrm{K}^{+}$离子 6 配位情况下半径为 $1.38 \AA(1 \AA=0.1 \mathrm{~nm})$, 明显大于 $\mathrm{Na}^{+}(6$ 配位, $1.02 \AA)$, 在 $\mathrm{Na}_{1-x} \mathrm{~K}_{x} \mathrm{Cl}$ 固溶体中, 随着 $x$ 增大, 晶胞参数和体积均呈现线性增大的趋势(图1(b) \& (c))。

使用Rietan FP软件, 在输入文本内输入 “ $\mathrm{NMODE}=1$ ” ，表示根据晶体结构进行X射线粉末衍 射谱的模拟计算。谱峰函数选择 “NPRFN $=1$ ” ，表示使用Toraya函数表示衍射峰的峰形。由于我们 只需要模拟图谱, 因此衍射的背景可忽略, 因此背景函数 “ $B K G D$ ” 全部设定为 0 。根据 $\mathrm{Na}_{1-x} \mathrm{~K}_{x} \mathrm{Cl}$ 中 $x$ 值的变化, 输入不同的结构参数进行模拟, 得到如图 $1(\mathrm{~d})$ 的衍射图谱。从模拟的图谱可见, 随着 $x$ 增 大, 晶胞参数变大 200 , 和 220 衍射往低角度偏移。这种表现形式非常直观, 可以让学生容易地接受 $X$ 射线衍射的相关知识, 深刻地认识到衍射峰位置与晶体结构的关系。

基于以上讲解, 结合现在的研究前沿, 如钻钛矿 $\mathrm{CsPbX}_{3}$ 光伏和发光材料, 可以给学生留下以下 课后思考题: (1) $\mathrm{CsPbCl}_{3}$ 和 $\mathrm{CsPbBr}_{3}$ 基本结构相同, 晶胞参数有微小差异, 哪个物质的晶胞参数较 大? 请给出原因。(2) $\mathrm{CsPbCl}_{3}$ 和 $\mathrm{CsPbBr}_{3}$, 这两种物质的衍射峰位置有什么关系? 

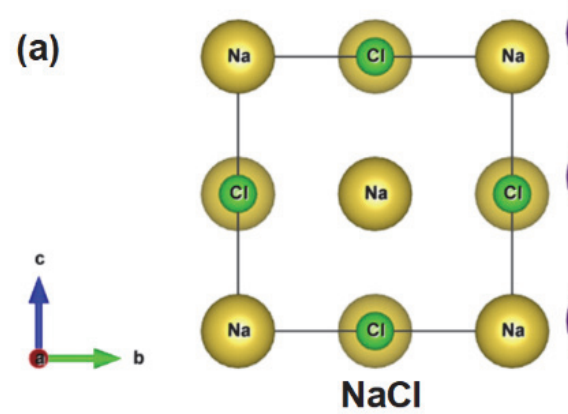
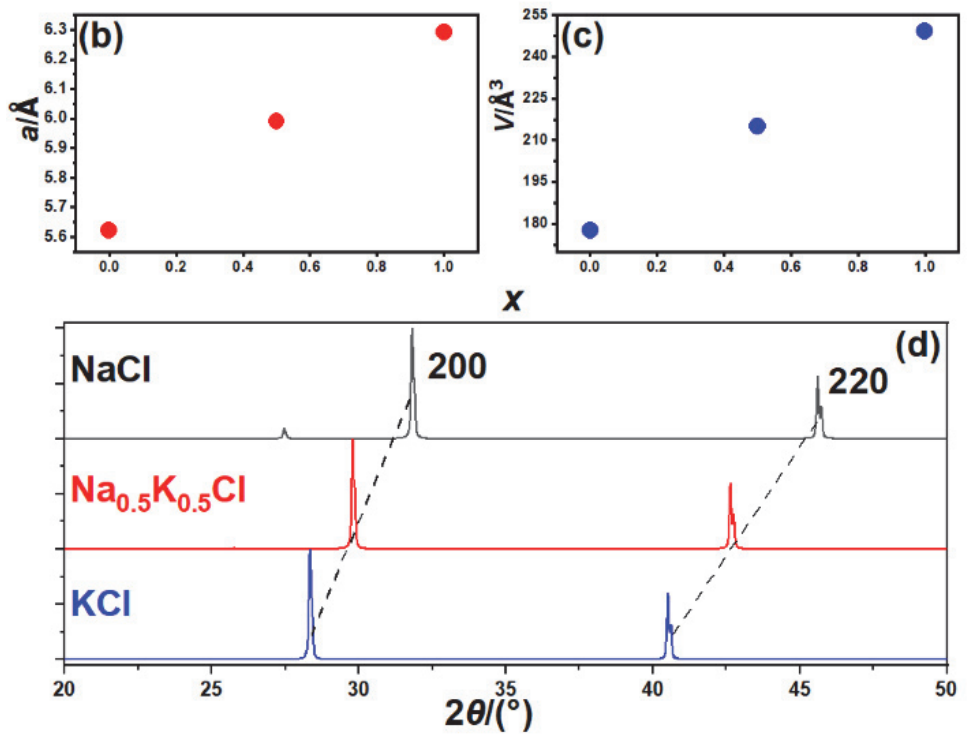

图1 (a) $\mathrm{NaCl}$ 和 $\mathrm{KCl}$ 的晶体结构图形, $\mathrm{Na}_{1-x} \mathrm{~K}_{x} \mathrm{Cl}$ 的 (b) $a$ 轴和 (c)体积参数, (d) $\mathrm{Na}_{1-x} \mathrm{~K}_{x} \mathrm{Cl}$ 的模拟 $\mathrm{X}$ 射线图谱

$1 \AA=0.1 \mathrm{~nm}$

\section{2 通过密度泛函理论计算模拟硅的拉曼散射光谱}

拉曼光谱基于入射激光与分子化学键振动相互作用, 发生非弹性碰撞, 激光损失一定的能量, 这个能量差与化学键振动频率对应, 所反映的信息可作为分子振动的指纹信息。实验中, 影响拉曼 光谱峰位和峰宽的因素非常多。影响峰位的因素有化学键的键强、晶体结构、温度和应力等, 影响 峰宽的因素有结晶度和温度等。对于拉曼光谱, 一般最受关注的是峰位。排除外界影响因素, 拉曼 峰位可粗略用以下公式描述:

$$
\text { 峰位 }\left(\mathrm{cm}^{-1}\right) \propto \sqrt{\frac{K}{m_{\mathrm{eff}}}}
$$

$K$ 为化学键力学常数, $m_{\mathrm{eff}}$ 为成键原子(离子)的有效质量:

$$
m_{\mathrm{eff}}=\frac{m_{1} \times m_{2}}{m_{1}+m_{2}}
$$

拉曼散射的产生条件是极化率的变化, 因此并不是所有振动都能产生相应的拉曼散射信号, 这 使得学生很难直观地建立晶体结构与拉曼散射的关系。另一方面, 物质内化学键的种类非常多, 使 得问题复杂化。基于这些原因, 仪器分析技术需要与密度泛函理论计算融合, 课程如此设计:

(1) 选择成分和结构相对简单的物质进行模拟, 这类物质的化学键种类尽量少, 降低学生理解问 题的难度; 
(2) 此物质的拉曼散射结果已被科研界熟知, 以便确认模拟结果的正确性;

(3) 此物质常用于拉曼散射实验;

(4) 模拟软件选取免费的Quantum Espresso (以下简称QE) 软件包, 可以完全避免版权纠纷, 只需 要引用原作者的论文即可 $(\mathrm{QE}$ 是意大利的里雅斯特国家模拟中心开发的软件包, 主要用于密度泛函 理论计算, 多个模块可以进行晶体结构优化、电子结构计算、介电函数计算光学性质、红外\&拉曼光 谱模拟、核磁共振模拟等) ${ }^{[5,6]}$ 。

根据以上设计理念，选择硅晶体，符合成分简单，化学键种类少(只有 $\mathrm{Si}-\mathrm{Si}$ 键)的原则。另外， 单晶硅常常用于拉曼光谱的校正, 其拉曼散射峰位于 $520-521 \mathrm{~cm}^{-1}$ 左右, 这方面知识已被广泛接受。 硅晶体为立方晶系, 空间群为 $F d \overline{3} m$, 每个单胞内含有 8 个硅原子, 每个硅原子在空间中等价(图2(a))。 硅与硅之间形成只有一类的化学键, 键长为 $2.35 \AA$ 。这里使用的是QE6.6版本进行密度泛函理论计 算。要计算拉曼光谱, 需要两个过程:

(1) 首先需要进行自洽场计算: 设定截断能 “ecutwfc” 为 $30 \mathrm{Ry}$ (约 $408 \mathrm{eV})$, 这个能量对于计算 纯硅足够高, 保证一定的计算精度; 自洽计算的收玫标准设定为 $10^{-6} \mathrm{Ry}$ 。 $K$ 点为 $2 \times 2 \times 2$; 由于软件 本身的限制, 如果后续需要计算拉曼光谱, 则规定使用局域梯度近似的模守恒赝势。

(2) 自洽场计算结束后, 在得到电子状态的基础上, 进行声子计算并导出拉曼光谱, 结果如图2(b) 所示。

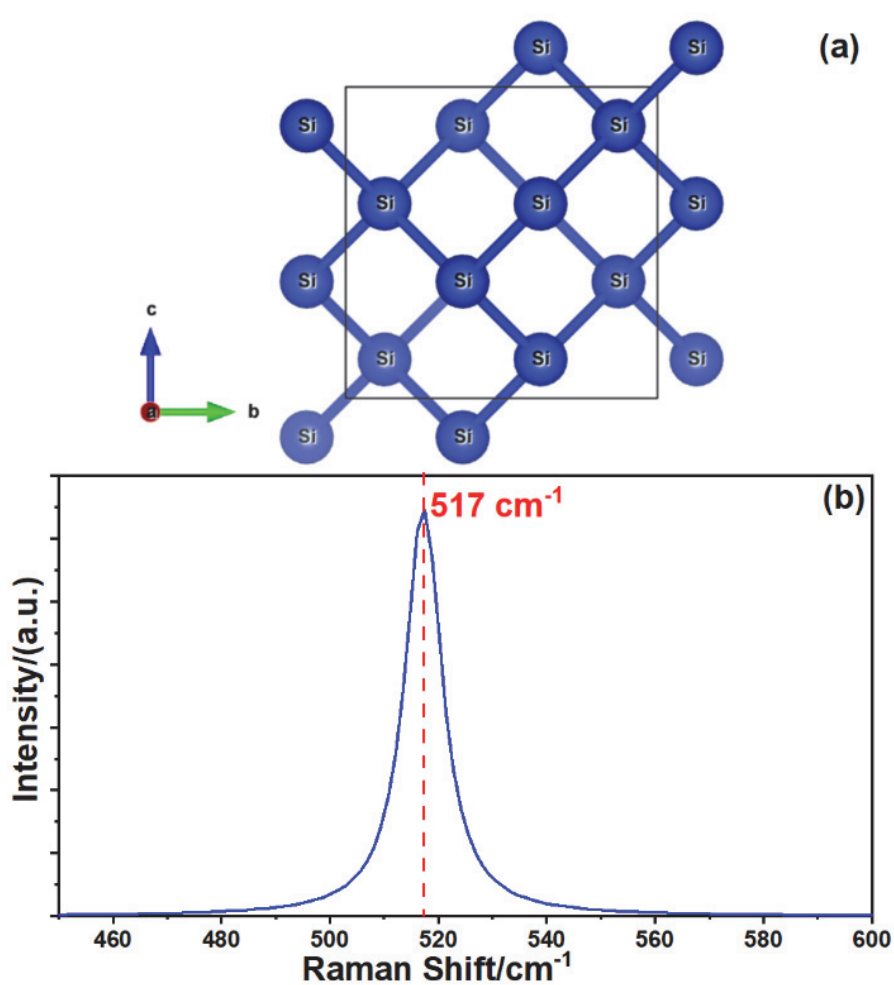

图2 (a) 立方晶硅的晶体结构; (b) 立方晶硅的模拟拉曼光谱

计算结果显示, 硅晶体的拉曼散射峰在 $517 \mathrm{~cm}^{-1}$, 第一性原理的计算结果与实验值 $520-521 \mathrm{~cm}^{-1}$ 仅有3-4 $\mathrm{cm}^{-1}$ 的微小差别, 表明模拟计算结果比较合理。现代分析技术课程包含理论授课和仪器实 操两部分, 模拟计算的部分属于理论授课, 但是计算结果可以穿插到实验的实操上面。每天拉曼测 试实验前都需要使用单晶硅做一次仪器校正, 现场实操的校正光谱, 可以与模拟计算的光谱进行比 对，加深学生对拉曼散射知识的印象。 
掺杂是材料改性的常用手段, 是物理、化学和材料等方向学生的学习重点。根据上述内容, 可 以给学生留下课后思考题: 碳和硅是同族元素, 如果碳掺杂进硅晶体, 部分替换了硅, 那么拉曼光 谱会发生什么变化？请解释原因。

\section{3 结语}

通过Rietan FP程序模拟X射线粉末衍射图谱, QE程序计算材料的拉曼光谱, 与现代分析技术课 程中的衍射和拉曼散射部分结合, 把抽象、难懂的微观原子级别现象以简单、直观、可视化的形式 表现出来并结合课程实操, 有助于让学生更深入地掌握现代仪器分析的知识和物质内部规律, 提高 学习效果, 为研究生将来成为相关专业的工程人员或科研人员夯实基础。此外, 模拟计算和密度泛 函理论计算与课程结合, 大大丰富了现代分析技术的教学方法。

\section{参 考 文 献}

[1] 张春芳, 张翠妙, 李江涛, 韩琳玉, 顾芳, 王海军. 大学化学, 2019, 34 (6), 83.

[2] 张鹏. 大学物理, 2021, 40 (6), 62 .

[3] 吴玉辉. 信息记录材料, 2019, 20 (9), 119.

[4] Izumi, F.; Momma, K. Solid State Phenom. 2007, 130, 15.

[5] Paolo, G.; Stefano, B.; Nicola, B.; Matteo, C.; Roberto, C.; Carlo, C.; Davide, C.; Guido, L. C.; Matteo, C.; Ismaila, D.; et al. J. Phys. Condens. Matter 2009, 21 (39), 395502.

[6] Giannozzi, P.; Andreussi, O.; Brumme, T.; Bunau, O.; Buongiorno Nardelli, M.; Calandra, M.; Car, R.; Cavazzoni, C.; Ceresoli, D.; Cococcioni, M.; et al. J. Phys. Condens. Matter 2017, 29 (46), 465901. 